\title{
Análise multivariada para seleção de clones de eucalipto destinados à produção de carvão vegetal
}

\author{
Ana Flávia Neves Mendes Castro(1), Renato Vinícius Oliveira Castro ${ }^{(2)}$, Angélica de Cássia Oliveira Carneiro(1), \\ João Eustáquio de Lima ${ }^{(3)}$, Rosimeire Cavalcante dos Santos ${ }^{(4)}$, Bárbara Luísa Corradi Pereira ${ }^{(1)}$ \\ e Isabel Cristina Nogueira Alves ${ }^{(5)}$
}

\begin{abstract}
(1)Universidade Federal de Viçosa (UFV), Departamento de Engenharia Florestal, Avenida P.H. Rolfs, s/no, Campus Universitário, CEP $36570-000$ Viçosa, MG. E-mail: mendesafn@hotmail.com, cassiacarneiro@ufv.br, babicorradi@gmail.com (2)Universidade de Brasília, Departamento de Engenharia Florestal, Campus Darcy Ribeiro, CEP 70904-970 Brasília, DF. E-mail: castrovo@ymail.com (3)UFV, Departamento de Economia Rural. E-mail: jelima@ufv.br ${ }^{(4)}$ Universidade Federal do Rio Grande do Norte, Unidade Acadêmica Especializada em Ciências Agrárias, CEP 59072-970 Natal, RN. E-mail: meire_caico@yahoo.com.br ${ }^{(5)}$ Universidade Federal de Lavras, Departamento de Ciência e Tecnologia da Madeira, CEP 37200-000 Lavras, MG. E-mail: isabel.alves@posgrad.ufla.br
\end{abstract}

Resumo - O objetivo deste trabalho foi avaliar a qualidade da madeira de clones de Eucalyptus spp. destinados à produção de carvão vegetal, estudar correlações entre propriedades da madeira e do carvão e identificar os clones com maior potencial para uso energético. Os dados de clones de 18 povoamentos, no Cerrado mineiro, foram submetidos às análises multivariada de correlação canônica; de componentes principais; e de agrupamento. As propriedades do carvão vegetal são fortemente correlacionadas às da madeira, principalmente a densidade aparente do carvão vegetal e o rendimento gravimétrico. Os materiais mais velhos e de maior densidade básica da madeira apresentam maior qualidade para uso energético. A análise de componentes principais é eficiente para ranquear os materiais quanto à qualidade da madeira, e o método de agrupamento é capaz de estratificar com sucesso os clones pela qualidade da madeira.

Termos para indexação: Eucalyptus, componentes principais, estatística multivariada, florestas energéticas, propriedades tecnológicas da madeira, rendimento em carvão.

\section{Multivariate analysis for the selection of eucalyptus clones destined for charcoal production}

\begin{abstract}
The objective of this work was to evaluate the wood quality of Eucalyptus spp. clones for the production of charcoal, to study the correlations between wood and charcoal properties, and to identify clones with the greatest potential for energy use. Data of clones from 18 settlements, in the Cerrado region of the state of Minas Gerais, Brazil, were subjected to the multivariate analysis of the canonical correlation analysis; principal component analysis; and cluster analysis. Charcoal properties are strongly correlated with the wood ones, mainly charcoal bulk density and gravimetric yield. Older materials with higher wood density have a higher quality for energy use. Principal component analysis is efficient to rank the materials as for wood quality, and the clustering method is able to successfully stratify clones by their wood quality.
\end{abstract}

Index terms: Eucalyptus, principal components, multivariate statistics, energy forests, wood technological properties, charcoal yield.

\section{Introdução}

As florestas de eucalipto têm sido as mais indicadas para fins energéticos, em razão de sua excelente produção volumétrica e da boa qualidade da madeira. Esse bom desempenho da cultura é em grande parte resultante do intenso melhoramento genético realizado com a espécie e do manejo adequado dos povoamentos (Goulart et al., 2003).

Com a alta competitividade do mercado, é importante que as empresas florestais busquem permanentemente por materiais genéticos mais produtivos e com propriedades da madeira adequadas para os diversos fins. Há muitos materiais genéticos melhorados para o aprimoramento das propriedades tecnológicas da madeira, com vistas à produção de carvão vegetal de melhor qualidade. No entanto, novas pesquisas ainda são necessárias para possibilitar o aprimoramento contínuo da espécie (Castro, 2011).

O carvão vegetal é superior à madeira quanto à produção de energia, em razão de sua maior concentração de carbono fixo e, consequentemente, 
de seu maior poder calorífico. No entanto, as propriedades do carvão vegetal são bastante variáveis, pois sofrem influência direta da matéria prima que lhe deu origem e do processo de carbonização, que são aspectos de difícil controle. São muitas as variáveis da madeira que influenciam a qualidade do carvão vegetal, como: a idade da madeira, a percentagem de cerne e alburno, o teor de extrativos, o teor de lignina total, entre outras. Madeiras mais velhas apresentam maior densidade e dão origem a carvão vegetal mais denso. Portanto, na escolha dos melhores materiais genéticos para a produção de carvão vegetal, deve-se considerar a densidade básica da madeira e a idade da árvore (Ferreira et al., 1979; Ribeiro \& Zani Filho, 1993; Castro, 2011). Essas características devem estar necessariamente associadas a alta produtividade, que é um caráter mais complexo e de obtenção mais difícil.

À medida que aumenta o número de variáveis a serem interpretadas, sua análise por meio da estatística univariada torna-se mais complexa. As análises multivariadas, inicialmente utilizadas apenas nas áreas de psicologia, ciências sociais e biologia (Trugilho et al., 2003), possuem grande aplicação nos casos em que diversos fatores influenciam o resultado, com diferentes ponderações (Mingoti, 2005).

O objetivo deste trabalho foi avaliar a qualidade da madeira de clones de Eucalyptus spp. destinados à produção de carvão vegetal, estudar correlações entre propriedades da madeira e do carvão, e indicar os clones com maior potencial para uso energético.

\section{Materiais e Métodos}

Os dados utilizados neste estudo provieram de plantios comerciais, pertencentes à Gerdau S.A, de clones não desbastados de Eucalyptus spp. (Tabela 1), localizados no Município de Lassance, no Norte de Minas Gerais.

A região de plantio encontra-se no bioma Cerrado, com clima do tipo Aw, conforme a classificação de Köppen, tropical chuvoso, caracterizado pela presença de invernos secos e verões chuvosos. Possui média anual de precipitação de $1.500 \mathrm{~mm}$, com variação de 750 a $2.000 \mathrm{~mm}$. As chuvas se concentram de outubro a março, e a temperatura média do mês mais frio é superior a $18^{\circ} \mathrm{C}$ (Ribeiro \& Walter, 1998).

Os solos predominantes são o Latossolos Vermelho e Vermelho-Amarelo, porosos, permeáveis e bem drenados. A textura é predominantemente arenosa ou areno-argilosa, com cerca de $25 \%$ de argila na camada de 0 a $40 \mathrm{~cm}$.

Para as análises da madeira e a obtenção do carvão vegetal, foram retirados de cada árvore cinco discos, nas alturas correspondentes a 0, 25, 50, 75 e 100\% da altura comercial do tronco (Castro et al., 2011; Pereira et al., 2012). Inicialmente, fez-se a medição das percentagens de cerne e alburno de cada disco, antes que as demais análises fossem realizadas. Essas percentagens foram determinadas com duas retas perpendiculares, que passavam sobre a medula. Obteve-se, então, a medida da distância das bordas até o início do cerne, bem como a medida do próprio cerne. A área do alburno foi calculada pela subtração da área de cerne da área total. A relação cerne/alburno foi calculada a partir de suas respectivas áreas.

Para a determinação da densidade básica da madeira, foram retiradas duas cunhas opostas, que passavam pela medula de todos os discos utilizados. Utilizou-se o método de imersão em água, descrito por Vital (1984). Os valores foram calculados a partir da média aritmética das densidades das respectivas cunhas. O restante dos discos foi seccionado, tendo-se destinado uma parte para a produção de carvão e outra para determinação da composição química e do poder calorífico superior das amostras compostas.

Tabela 1. Identificação dos materiais genéticos de Eucalyptus spp. utilizados, com suas respectivas idades por ocasião das avaliações.

\begin{tabular}{lccc}
\hline Material & Clone & Idade (anos) & Espécie \\
\hline 1 & GG 100 & 3 & E. grandis $\times$ E. urophylla \\
2 & GG 100 & 4 & E. grandis $\times$ E. urophylla \\
3 & GG 100 & 5 & E. grandis $\times$ E. urophylla \\
4 & GG 100 & 7 & E. grandis $\times$ E. urophylla \\
5 & GG 157 & 3 & E. urophylla \\
6 & GG 157 & 4 & E. urophylla \\
7 & GG 157 & 5 & E. urophylla \\
8 & GG 157 & 7 & E. urophylla \\
9 & GG 680 & 3 & E. grandis $\times$ E. urophylla \\
10 & GG 680 & 4 & E. grandis $\times$ E. urophylla \\
11 & GG 680 & 5 & E. grandis $\times$ E. urophylla \\
12 & GG 680 & 7 & E. grandis $\times$ E. urophylla \\
13 & GG 3616 & 7 & E. camaldulensis \\
14 & GG 3403 & 7 & E. urophylla \\
15 & GG 3370 & 7 & E. grandis \\
16 & GG 3192 & 7 & E. urophylla \\
17 & GG 3520 & 7 & E. urophylla \\
18 & GG 3119 & 7 & E. camaldulensis \\
\hline
\end{tabular}


Para a análise química e a determinação do poder calorífico da madeira, as amostras foram transformadas em serragem, com moinho de laboratório tipo Wiley, de acordo com a norma T $257 \mathrm{~cm}-85$ (Tappi Technical Divisions and Committees, 1985). As análises foram feitas com a fração que passou pela peneira $\mathrm{n}^{-} 16$ internacional (40 mesh) e ficou retida na peneira $\mathrm{n}^{\mathrm{o}} 24$ internacional (60 mesh). As amostras foram secas em estufa a $103 \pm 2^{\circ} \mathrm{C}$, até a obtenção de massa constante. $O$ poder calorífico superior da madeira foi determinado de acordo com a metodologia descrita na norma NBR 8633 (Associação Brasileira de Normas Técnicas, 1984), tendo-se utilizado uma bomba calorimétrica adiabática. Para a análise química, determinou-se o teor absolutamente seco (a.s.) da madeira e aplicou-se a norma T $264 \mathrm{~cm}-97$ (Tappi Technical Divisions and Committees, 1997).

Os teores de extrativos na madeira foram determinados em duplicata, de acordo com a norma TAPPI $264 \mathrm{~cm}-88$, com uso do método de extrativos totais, tendo-se utilizado o etanol/benzeno ao invés do etanol/tolueno. Os teores de lignina insolúvel foram determinados em duplicata, pelo método Klason, modificado de acordo com o procedimento proposto por Gomide \& Demuner (1986), derivado da norma TAPPI T $222 \mathrm{~cm}-88$. A lignina solúvel foi determinada por espectrometria, conforme Goldschimid (1971), a partir da diluição do filtrado proveniente do procedimento realizado para obtenção da lignina insolúvel, e o teor de lignina total foi obtido com a soma dos dois valores. O teor de holocelulose foi obtido pelo somatório dos teores de extrativos e lignina totais subtraído de 100.

$\mathrm{O}$ valor da relação siringil/guaiacil $(\mathrm{SG})$ da lignina foi determinado, em duplicata, por meio da cromatografia líquida, após oxidação da serragem da madeira com nitrobenzeno, conforme adaptações no método descrito por Lin \& Dence (1992). Duzentos miligramas a.s. de serragem livre de extrativo foram colocados em reatores de aço inox, juntamente com $7 \mathrm{~mL}$ da solução aquosa de $\mathrm{NaOH}\left(2 \mathrm{~mol} \mathrm{~L}^{-1}\right)$ e $0,5 \mathrm{~mL}$ de nitrobenzeno. Após lacrar os reatores, a amostra foi levada ao banho maria com glicerina, por duas horas e trinta minutos, a $170^{\circ} \mathrm{C}$. Em seguida, o material oxidado foi transferido para um funil de separação e extraído com clorofórmio, por cinco vezes, com uso de $30 \mathrm{~mL}$ do solvente em cada extração. Após a primeira extração, foram adicionados $3 \mathrm{~mL}$ de $\mathrm{HCl}$ (4 mol L-1) na fase aquosa. As fases orgânicas foram reunidas e o solvente evaporado em capela. A amostra foi transferida para um balão volumétrico de $50 \mathrm{~mL}$ e o volume completado com solução de acetonitrila/água $(1: 1 \mathrm{v} / \mathrm{v})$. Em seguida, a solução resultante foi filtrada em membrana de celulose regenerada de $0,45 \mathrm{~m}$ e analisada por cromatografia líquida de alta eficiência (Clae). A separação dos produtos da oxidação por nitrobenzeno foi alcançada com uma coluna LC-18. A fase móvel usada foi acetonitrila/água $(1: 6 \mathrm{v} / \mathrm{v})$, com $\mathrm{pH}$ igual a 2,6, tamponado com ácido trifluoroacético (TFA), com detecção feita a: UV, $280 \mathrm{~nm} ; \mathrm{T}, 40^{\circ} \mathrm{C}$; fluxo, 1,5 mL por minuto; e injeção de $20 \mu \mathrm{L}$. O padrão cromatográfico foi de vanilina para guaiacil e siringaldeído para siringil. A pressão utilizada foi de, aproximadamente, $160 \mathrm{kgf} \mathrm{cm}^{-2}$.

A carbonização foi feita com as amostras de madeira retiradas dos discos. Para cada árvore, obteve-se uma amostra composta. As amostras foram, então, secas em estufa a $103 \pm 2^{\circ} \mathrm{C}$, até a obtenção de massa constante. As carbonizações foram realizadas em mufla de laboratório, com aquecimento elétrico, com as amostras inseridas em um contêiner metálico. O tempo total de carbonização da madeira foi de cinco horas, com taxa de aquecimento média de $1,56^{\circ} \mathrm{C} \mathrm{min}^{-1}$. A temperatura inicial foi de $30^{\circ} \mathrm{C}$, e a final, de $450^{\circ} \mathrm{C}$ - estabilizada por um período de 30 minutos. Após as carbonizações, foi determinado o rendimento gravimétrico em carvão, com base na massa de matéria seca da madeira.

Para a análise química imediata do carvão vegetal, as amostras foram moídas e peneiradas a uma granulometria de aproximadamente $0,2 \mathrm{~mm}$, conforme a NBR 8112 (Associação Brasileira de Normas Técnicas, 1986), com adaptações na determinação dos teores de materiais voláteis, cinzas e carbono fixo. O teor de materiais voláteis foi determinado pelo aquecimento do carvão a $950^{\circ} \mathrm{C}$, em forno mufla, com as amostras tampadas em cadinhos. As amostras foram inicialmente levadas à porta da mufla por dois minutos, para aclimatação, e ficaram por mais 9 min no interior da mufla. O teor de cinzas foi determinado após a combustão completa do carvão, em mufla a $650^{\circ} \mathrm{C}$, durante seis horas. A massa residual em relação à massa de carvão seco foi considerada como o teor de cinzas. O teor de carbono fixo foi calculado pela soma dos teores de materiais voláteis e cinzas, subtraída de 100.

A densidade relativa aparente do carvão foi determinada de acordo com o método proposto por 
Vital (1984), com uso de balança hidrostática, para a determinação do volume deslocado. As amostras de carvão foram pesadas e, posteriormente, imersas em água para a determinação do volume deslocado.

O poder calorífico superior do carvão e o poder calorífico superior da madeira foram determinados de acordo com a metodologia descrita pela norma NBR 8633 (Associação Brasileira de Normas Técnicas, 1984), com uso de bomba calorimétrica adiabática.

As análises de correlação canônica, de componentes principais e de agrupamento foram realizadas pelo programa Stata 11. A partir dos valores de correlação canônica, resumiram-se as informações do conjunto de variáveis das propriedades da madeira dos 18 materiais avaliados, a partir de combinações lineares, a fim de maximizar a correlação com o conjunto de propriedades do carvão vegetal originado desses materiais.

A variável SG não entrou no cálculo da correlação canônica, nem nas demais análises, uma vez que essa variável correlaciona-se com o teor de lignina total, o que poderia interferir no resultado se ambas as variáveis fossem analisadas juntas.

As combinações lineares construídas foram chamadas de variáveis canônicas, enquanto a correlação entre elas foi chamada de correlação canônica. Essa correlação mede basicamente o grau de associação existente entre os dois conjuntos de variáveis (Mingoti, 2005). Buscaram-se combinações lineares que possibilitavam a maximização da correlação entre elas. O primeiro par canônico foi aquele com a maior correlação entre as variáveis canônicas. Em seguida, foram calculadas outras duas variáveis canônicas - outro par canônico - com a condição de serem ortogonais às primeiras e terem o máximo coeficiente de correlação. O segundo par, portanto, apresentou a segunda maior correlação entre as variáveis canônicas, e assim sucessivamente. O coeficiente de correlação canônica utilizado foi o valor absoluto do coeficiente de correlação de Pearson, entre as variáveis canônicas de cada par canônico, conforme sugerido por Mingoti (2005).

Confirmada a correlação canônica, buscou-se ranquear os clones conforme suas propriedades de madeira, por meio da análise dos componentes principais (ACP), de forma a identificar indiretamente os melhores materiais genéticos para a produção de carvão vegetal de alta qualidade. Pela ACP, pode-se explicar a estrutura de variância e covariância de um vetor aleatório (composto de p-variáveis aleatórias), por meio da construção de combinações lineares das variáveis originais. Com $\mathrm{p}$ variáveis originais, p componentes principais foram obtidos. Em geral, deseja-se diminuir o número de variáveis a serem avaliadas e facilitar a interpretação das combinações lineares construídas. A obtenção das componentes principais envolve a decomposição da matriz de covariâncias do vetor aleatório de interesse. Uma vez determinados os componentes principais, seus escores (valores) foram calculados para cada elemento amostral. Deste modo, os valores de cada componente puderam ser analisados com técnicas estatísticas usuais, como análise de variância, análise de regressão, ou análise de agrupamento (Mingoti, 2005).

Com os escores dos componentes principais, a análise de agrupamento permite classificar o conjunto de unidades ou observações em grupos relativamente homogêneos. Este método permite determinar, a partir das características das unidades, subconjuntos em que cada unidade pertença somente a um subconjunto, e que as unidades agrupadas em um mesmo subconjunto sejam similares entre si e diferentes das unidades de outros subconjuntos (Souza, 2000).

O uso de técnicas hierárquicas aglomerativas é amplamente difundido na análise de agrupamento. Essas técnicas permitem a classificação dos indivíduos mediante sucessivas fusões dos $\mathrm{n}$ indivíduos em grupos. O procedimento básico consiste em computar uma matriz de distância ou de similaridade entre os indivíduos, a partir da qual inicia-se um processo de sucessivas fusões, com base na proximidade ou similaridade entre eles. Os resultados desse processo são passíveis de representação em dendrograma, que nada mais é do que um diagrama bidimensional, que exibe as fusões realizadas em cada nível e culmina no estágio em que todos os indivíduos estão num único grupo (Souza \& Lima, 2003).

\section{Resultados e Discussão}

Com base nos valores médios (Tabela 2) obtidos para as propriedades da madeira (Conjunto 1) e do carvão vegetal (Conjunto 2), obteve-se a correlação canônica entre esses conjuntos, com geração inicial de seis pares canônicos. Entretanto, somente os três primeiros pares canônicos apresentaram coeficientes de correlação significativos e foram utilizados na análise: 
$\mathrm{U}_{1}=-1,0882$ Idade $+0,6155 \mathrm{CA}+1,3233 \mathrm{DBM}+$ 0,3504PCSM - 0,4531EXT + 0,1288LT e

$\mathrm{V}_{1}=1,5164 \mathrm{RGCV}+0,2071 \mathrm{PCSC}+0,7974 \mathrm{DC}-$ 10,5267VOL - 1,1383CZ - 9,2263TCF, com $\mathrm{r}_{\mathrm{U}_{1} \mathrm{~V}_{1}}=0,9937$;

$\mathrm{U}_{2}=0,6746$ Idade - 0,1607CA - 1,7011DBM $0,0747 \mathrm{PCSM}+0,1622 \mathrm{EXT}-0,0191 \mathrm{LT}$ e

$\mathrm{V}_{2}=0,7980 \mathrm{RGCV}+0,1818 \mathrm{PCSC}+1,0387 \mathrm{DC}+$ $6,8548 \mathrm{VOL}+1,6794 \mathrm{CZ}+5,2799 \mathrm{TCF}$,

com $\mathrm{r}_{\mathrm{U}_{2} \mathrm{v}_{2}}=0,9673$; e

$\mathrm{U}_{3}=-0,2478 \mathrm{Idade}+0,3458 \mathrm{CA}+0,2198 \mathrm{DBM}-$ 0,0105PCSM - 0,3636EXT - 1,1050LT e

$\mathrm{V}_{3}=0,4743 \mathrm{RGCV}-0,0284 \mathrm{PCSC}+0,4835 \mathrm{DC}+$ $144,0054 \mathrm{VOL}+33,0346 \mathrm{CZ}+119,0237 \mathrm{TCF}$,

com $\mathrm{r}_{\mathrm{U}_{3} \mathrm{~V}_{3}}=0,8323$;

em que: a idade é expressa em anos; CA é a relação cerne/alburno; DBM é a densidade básica da madeira $\left(\mathrm{g} \mathrm{cm}^{-3}\right)$; PCSM é o poder calorífico superior da madeira ( $\mathrm{kcal} \mathrm{kg}^{-1}$ ); EXT é a percentagem de extrativos da madeira; LT é o teor de lignina total (\%); RGCV é o rendimento gravimétrico em carvão vegetal (\%);
PCSC é o poder calorífico superior do carvão vegetal ( $\left.\mathrm{kcal} \mathrm{kg}^{-1}\right)$; DC é a densidade aparente do carvão $\left(\mathrm{g} \mathrm{cm}^{-3}\right)$; VOL refere-se ao teor de voláteis (\%); CZ é o teor de cinzas (\%); e TCF, é o teor de carbono fixo (\%); $\mathrm{r}_{\mathrm{U}_{\mathrm{i}} \mathrm{v}_{\mathrm{i}}}$ é a correlação canônica entre as características da madeira e do carvão vegetal.

Na Tabela 3, estão apresentados os coeficientes de correlação entre as variáveis originais de propriedades da madeira e as variáveis canônicas das combinações lineares das variáveis de madeira, bem como a correlação entre as variáveis originais das características do carvão vegetal e as variáveis canônicas das combinações lineares das variáveis de carvão vegetal. Com base nos pares canônicos apresentados na Tabela 3, observouse predomínio de idade, CA e EXT, entre as variáveis que compõem o primeiro par canônico do conjunto 1 (características da madeira). Para as variáveis que compõem o conjunto 2 (características do carvão vegetal), a variável rendimento gravimétrico em carvão vegetal (RGCV) teve predomínio absoluto.

No segundo par canônico, houve predomínio da variável idade, seguida da DBM e do PCS, no

Tabela 2. Médias das propriedades de madeira (conjunto 1) e propriedades do carvão vegetal (conjunto 2).

\begin{tabular}{|c|c|c|c|c|c|c|c|c|c|c|c|c|}
\hline \multirow[t]{2}{*}{ Material } & \multirow[t]{2}{*}{ Idade } & \multicolumn{5}{|c|}{ Conjunto 1} & \multicolumn{6}{|c|}{ Conjunto 2} \\
\hline & & $\mathrm{CA}$ & DBM & PCSM & EXT & LT & RGCV & PCSC & $\mathrm{DC}$ & VOL & $\mathrm{CZ}$ & TCF \\
\hline 1 & 3 & 1,16 & 0,45 & 4542,47 & 2,37 & 32,23 & 32,98 & 7409,17 & 0,30 & 21,34 & 1,24 & 77,43 \\
\hline 2 & 4 & 1,48 & 0,46 & 4654,23 & 4,08 & 31,46 & 33,10 & 7477,67 & 0,31 & 21,57 & 0,89 & 77,55 \\
\hline 3 & 5 & 1,45 & 0,46 & 4718,88 & 3,90 & 30,82 & 33,18 & 7446,33 & 0,26 & 23,21 & 0,96 & 75,83 \\
\hline 4 & 7 & 1,61 & 0,52 & 4617,17 & 4,68 & 32,86 & 33,53 & 7332,17 & 0,33 & 23,77 & 0,48 & 75,75 \\
\hline 5 & 3 & 1,81 & 0,45 & 4619,52 & 2,91 & 31,20 & 32,61 & 7278,67 & 0,32 & 22,05 & 1,44 & 76,52 \\
\hline 6 & 4 & 1,38 & 0,47 & 4718,15 & 3,04 & 30,73 & 34,29 & 7313,67 & 0,29 & 22,42 & 0,96 & 76,63 \\
\hline 7 & 5 & 1,40 & 0,53 & 4687,15 & 3,94 & 32,11 & 34,49 & 7343,17 & 0,34 & 24,35 & 0,55 & 75,10 \\
\hline 8 & 7 & 1,77 & 0,54 & 4614,77 & 3,33 & 31,69 & 34,81 & 7382,67 & 0,32 & 25,40 & 0,35 & 74,25 \\
\hline 9 & 3 & 0,96 & 0,49 & 4586,44 & 4,02 & 32,04 & 34,31 & 7331,00 & 0,34 & 23,13 & 0,51 & 76,36 \\
\hline 10 & 4 & 1,14 & 0,50 & 4621,15 & 3,50 & 31,63 & 33,91 & 7363,33 & 0,33 & 23,89 & 0,65 & 75,46 \\
\hline 11 & 5 & 1,60 & 0,53 & 4480,26 & 3,44 & 31,32 & 34,65 & 7305,50 & 0,34 & 24,97 & 0,32 & 74,71 \\
\hline 12 & 7 & 1,85 & 0,56 & 4481,65 & 4,31 & 33,82 & 34,96 & 7282,17 & 0,35 & 24,05 & 0,17 & 75,77 \\
\hline 13 & 7 & 0,88 & 0,53 & 4557,67 & 4,30 & 29,94 & 31,24 & 7413,10 & 0,36 & 26,72 & 0,35 & 72,93 \\
\hline 14 & 7 & 0,60 & 0,55 & 4551,00 & 4,97 & 31,79 & 31,47 & 7502,13 & 0,37 & 24,76 & 0,33 & 74,91 \\
\hline 15 & 7 & 0,79 & 0,57 & 4549,83 & 4,15 & 29,10 & 31,86 & 7490,11 & 0,40 & 25,79 & 0,35 & 73,86 \\
\hline 16 & 7 & 0,86 & 0,55 & 4568,33 & 4,70 & 28,18 & 32,18 & 7069,66 & 0,36 & 25,74 & 0,41 & 73,86 \\
\hline 17 & 7 & 0,59 & 0,59 & 4506,83 & 4,77 & 29,94 & 31,44 & 7616,30 & 0,39 & 26,08 & 0,36 & 73,56 \\
\hline 18 & 7 & 0,50 & 0,57 & 4595,33 & 3,10 & 30,09 & 30,90 & 7547,14 & 0,42 & 24,99 & 0,64 & 74,36 \\
\hline Média & & 1,21 & 0,52 & 4592,82 & 3,86 & 31,16 & 33,11 & 7383,55 & 0,34 & 24,12 & 0,61 & 75,27 \\
\hline CV (\%) & & 36,46 & 8,82 & 1,56 & 18,82 & 4,35 & 4,06 & 1,67 & 11,66 & 6,60 & 57,60 & 1,76 \\
\hline
\end{tabular}

Idade, em anos; CA, relação cerne/alburno; DBM, densidade básica da madeira $\left(\mathrm{g} \mathrm{cm}^{-3}\right)$; PCSM, poder calorífico superior da madeira $\left(\mathrm{kcal} \mathrm{kg}^{-1}\right)$; EXT, teor de extrativos da madeira (\%); LT, teor de lignina total (\%); RGCV, rendimento gravimétrico em carvão vegetal (\%); PCSC, poder calorífico superior do carvão vegetal ( $\left.\mathrm{kcal} \mathrm{kg}^{-1}\right)$; DC, densidade aparente do carvão $\left(\mathrm{g} \mathrm{cm}^{-3}\right)$; VOL, teor de voláteis (\%); CZ, teor de cinzas (\%); e TCF, teor de carbono fixo (\%). 
conjunto 1. Além destas, destacaram-se as correlações entre DC, VOL, CZ e TCF para o conjunto 2 .

No terceiro par canônico, houve alta correlação apenas para a variável LT, do conjunto1.

As variáveis canônicas $U_{1}, U_{2}$ e $U_{3}$, que representam as características de madeira, explicaram 28, 29 e $15 \%$ da variância total observada, respectivamente. Portanto, essas três variáveis canônicas explicaram $71 \%$ da variância total. Para as variáveis canônicas V1, V2 e V3, que representam as características do carvão vegetal, as variâncias explicaram, respectivamente, 23,39 e $8 \%$ da variância total, num total de $70 \%$ de variância explicada.

As variáveis canônicas de carvão vegetal correlacionaram-se significativamente com as variáveis de qualidade da madeira: idade, DBM, CA e EXT (Tabela 4). É interessante mencionar que estas variáveis foram justamente as que predominaram no conjunto 1 (Tabela 3 ).

A densidade básica da madeira (DBM) pode ser considerada como um parâmetro referencial, para a

Tabela 3. Correlação entre as variáveis originais da madeira (conjunto 1) e as variáveis canônicas das combinações lineares das variáveis de madeira $\left(\mathrm{U}_{\mathrm{i}}\right)$, e correlação entre as variáveis originais do carvão vegetal (conjunto 2) e as variáveis canônicas das combinações lineares das variáveis de carvão $\left(\mathrm{V}_{\mathrm{i}}\right)$

\begin{tabular}{|c|c|c|c|c|c|c|}
\hline Variável & $\mathrm{U}_{1}$ & $\mathrm{~V}_{1}$ & $\mathrm{U}_{2}$ & $\mathrm{~V}_{2}$ & $\mathrm{U}_{3}$ & $\mathrm{~V}_{3}$ \\
\hline \multicolumn{7}{|c|}{ Conjunto 1} \\
\hline Idade & $\underline{-0,5892^{(1)}}$ & - & $\underline{-0,6017}$ & - & $-0,0868$ & - \\
\hline $\mathrm{CA}$ & $\underline{0,6361}$ & - & 0,3414 & - & $-0,2202$ & - \\
\hline DBM & $-0,3763$ & - & $\underline{-0,9117}$ & - & $-0,0440$ & - \\
\hline PCSM & 0,2595 & - & $\underline{0.5537}$ & - & 0,1336 & - \\
\hline EXT & $\underline{-0,6892}$ & - & $-0,2833$ & - & $-0,3568$ & - \\
\hline LT & 0,4807 & - & 0,1483 & - & $\underline{-0,8471}$ & - \\
\hline \multicolumn{7}{|c|}{ Conjunto 2} \\
\hline RGCV & - & $\underline{0,7468}$ & - & 0,0813 & - & $-0,3861$ \\
\hline PCSC & - & $-0,2363$ & - & $-0,1670$ & - & $-0,1580$ \\
\hline DC & - & $-0,3830$ & - & $\underline{-0,8322}$ & - & 0,1090 \\
\hline VOL & - & $-0,4818$ & - & $\underline{-0,7457}$ & - & 0,2172 \\
\hline $\mathrm{CZ}$ & - & 0,3618 & - & $\underline{0,7573}$ & - & 0,3377 \\
\hline TCF & - & 0,4811 & - & $\underline{0,6950}$ & - & $-0,3470$ \\
\hline
\end{tabular}

${ }^{(1)}$ Valores sublinhados referem-se a correlações significativas a $5 \%$ e representam as variáveis que predominaram na explicação da variância total. Idade, em anos; CA, relação cerne/alburno; DBM, densidade básica da madeira $\left(\mathrm{g} \mathrm{cm}^{-3}\right)$; PCSM, poder calorífico superior da madeira $\left(\mathrm{kcal} \mathrm{kg}^{-1}\right)$; EXT, teor de extrativos da madeira (\%); LT, teor de lignina total (\%); RGCV, rendimento gravimétrico em carvão vegetal (\%); PCSC, poder calorífico superior do carvão vegetal $\left(\mathrm{kcal} \mathrm{kg}^{-1}\right)$; $\mathrm{DC}$, densidade aparente do carvão $\left(\mathrm{g} \mathrm{cm}^{-3}\right)$; VOL, teor de voláteis (\%); CZ, teor de cinzas (\%); e TCF, teor de carbono fixo $(\%)$ seleção de espécies florestais para produção de energia (Santos, 2010). Isso ocorre por ela depender de uma complexa combinação entre os constituintes anatômicos da madeira (Panshin \& Zeeuw, 1980). Portanto, a DBM fornece várias informações sobre a madeira, pois está relacionada a várias outras propriedades desta, o que a torna um importante parâmetro de qualificação da madeira, utilizado nos diversos segmentos da atividade industrial (Silva et al., 2004; Pereira et al., 2012).

Os resultados apresentados no presente trabalho confirmam, também, a importância da idade das árvores para o aumento da qualidade da madeira com vistas à produção de carvão vegetal. $\mathrm{O}$ envelhecimento das árvores promove transformações na madeira, como aumento da DBM e da proporção do cerne (Ferreira et al., 1979; Ribeiro \& Zani Filho, 1993). Como as características da madeira variam com o incremento da idade, espera-se que as características do carvão vegetal originado desse material também variem. Em geral, as madeiras com maior DBM originaram de carvão mais denso, o que está de acordo com o esperado.

Tabela 4. Correlação entre as características das madeiras (conjunto 1) e as variáveis canônicas das combinações lineares das variáveis de carvão vegetal $\left(\mathrm{V}_{\mathrm{i}}\right)$, e correlação entre as características do carvão vegetal (conjunto 2) e as variáveis canônicas das combinações lineares das variáveis de madeira $\left(\mathrm{U}_{\mathrm{i}}\right)$.

\begin{tabular}{lcccrcc}
\hline Variável & $\mathrm{U}_{1}$ & $\mathrm{~V}_{1}$ & $\mathrm{U}_{2}$ & $\mathrm{~V}_{2}$ & $\mathrm{U}_{3}$ & $\mathrm{~V}_{3}$ \\
\hline Conjunto 1 & & & & & & \\
Idade & - & $\underline{-0,5855^{(1)}}$ & - & $-0,5820$ & - & $-0,0723$ \\
CA & - & $\underline{\underline{0,6321}}$ & - & 0,3302 & - & $-0,1833$ \\
DBM & - & $-0,3739$ & - & $\underline{-0,8819}$ & - & $-0,0366$ \\
PCSM & - & 0,2579 & - & $\underline{0,5356}$ & - & 0,1112 \\
EXT & - & $\underline{-0,6849}$ & - & $-0,2741$ & - & $-0,2970$ \\
LT & - & 0,4776 & - & 0,1435 & - & $\underline{-0,7050}$ \\
\hline
\end{tabular}

\begin{tabular}{lrlrlrl}
\hline Conjunto 2 & & & & & & \\
RGCV & $\underline{0,7421}$ & - & 0,0786 & - & $-0,3213$ & - \\
PCSC & $-0,2348$ & - & $-0,1616$ & - & $-0,1315$ & - \\
DC & $-0,3805$ & - & $\underline{-0,8050}$ & - & 0,0908 & - \\
VOL & $-0,4788$ & - & $\underline{-0,7213}$ & - & 0,1807 & - \\
CZ & 0,3596 & - & $\underline{0,7325}$ & - & 0,2811 & - \\
TCF & 0,4780 & - & $\underline{0,6723}$ & - & $-0,2888$ & - \\
\hline
\end{tabular}

(1)Valores sublinhados referem-se a correlações significativas a 5\% e representam as variáveis que predominaram na explicação da variância total. Idade, em anos; CA, relação cerne/alburno; DBM, densidade básica da madeira $\left(\mathrm{g} \mathrm{cm}^{-3}\right)$; PCSM, poder calorífico superior da madeira $\left(\mathrm{kcal} \mathrm{kg}^{-1}\right)$; EXT, teor de extrativos da madeira (\%); LT, teor de lignina total (\%); RGCV, rendimento gravimétrico em carvão vegetal (\%); PCSC, poder calorífico superior do carvão vegetal $\left(\mathrm{kcal} \mathrm{kg}^{-1}\right)$; $\mathrm{DC}$, densidade aparente do carvão $\left(\mathrm{g} \mathrm{cm}^{-3}\right)$; VOL, teor de voláteis (\%); CZ, teor de cinzas (\%); e TCF, teor de carbono fixo (\%). 
Em trabalho semelhante, Protásio et al. (2013) utilizou técnicas de estatística multivariada e univariada na seleção e avaliação de clones de Eucalyptus para a produção de carvão vegetal e bioenergia. Os autores utilizaram variáveis físicas e químicas de madeira e de carvão vegetal e conseguiram agrupar clones com características da madeira similares. De acordo com os autores, dois pares canônicos explicaram $80 \%$ da variância total dos dados, com maior importância das variáveis lignina total, poder calorífico superior e teor de enxofre.

Dois dos componentes principais apresentaram raiz característica maior do que um (Tabela 5). Os escores desses dois componentes, obtidos a partir da matriz de correlações, representaram $72 \%$ da variância total dos dados (51 e $21 \%$, respectivamente) e foram utilizados para a análise de agrupamento (Figura 1). Três grupos foram formados da seguinte maneira: o primeiro, pelos materiais $14,17,16,15,18$ e 13 ; o segundo, apenas pelo material 12; e o terceiro pelos materiais $11,8,4$, $6,3,10,9,7,2,5$ e 1 .

Tabela 5. Agrupamento dos clones quanto à qualidade de madeira para produção de carvão vegetal, a partir da análise de componentes principais e do agrupamento pelo método de ligação simples, com base na distância euclidiana.

\begin{tabular}{|c|c|c|c|c|c|c|c|c|}
\hline \multirow{2}{*}{\multicolumn{3}{|c|}{ Grupo Ranque Material }} & \multicolumn{6}{|c|}{ Características da madeira } \\
\hline & & & Idade & CA & DBM & PCSM & EXT & LT \\
\hline \multirow{6}{*}{1} & $1^{\circ}$ & 17 & 7 & 0,59 & 0,59 & 4506,83 & 4,77 & $29,94(2,70)^{(1)}$ \\
\hline & $2^{\circ}$ & 16 & 7 & 0,86 & 0,55 & 4568,33 & 4,70 & $28,18(2,33)$ \\
\hline & $3^{\circ}$ & 15 & 7 & 0,79 & 0,57 & 4549,83 & 4,15 & $29,10(2,75)$ \\
\hline & $4^{\circ}$ & 14 & 7 & 0,60 & 0,55 & 4551,00 & 4,97 & $31,79(2,95)$ \\
\hline & $5^{\circ}$ & 13 & 7 & 0,88 & 0,53 & 4557,67 & 4,30 & $29,94(2,75)$ \\
\hline & $6^{\circ}$ & 18 & 7 & 0,50 & 0,57 & 4595,33 & 3,10 & $30,09(2,35)$ \\
\hline 2 & $7^{\circ}$ & 12 & 7 & 1,85 & 0,56 & 4481,65 & 4,31 & $33,82(2,40)$ \\
\hline \multirow{11}{*}{3} & $8^{\circ}$ & 4 & 7 & 1,61 & 0,52 & 4617,17 & 4,68 & $32,86(2,30)$ \\
\hline & $9^{\circ}$ & 11 & 5 & 1,60 & 0,53 & 4480,26 & 3,44 & $31,32(2,40)$ \\
\hline & $10^{\circ}$ & 8 & 7 & 1,77 & 0,54 & 4614,77 & 3,33 & $31,69(2,82)$ \\
\hline & $11^{\circ}$ & 7 & 5 & 1,40 & 0,53 & 4687,15 & 3,94 & $32,11(2,68)$ \\
\hline & $12^{\circ}$ & 9 & 3 & 0,96 & 0,49 & 4586,44 & 4,02 & $32,04(2,52)$ \\
\hline & $13^{\circ}$ & 10 & 4 & 1,14 & 0,50 & 4621,15 & 3,50 & $31,63(2,52)$ \\
\hline & $14^{\circ}$ & 3 & 5 & 1,45 & 0,46 & 4718,88 & 3,90 & $30,82(2,53)$ \\
\hline & $15^{\circ}$ & 2 & 4 & 1,48 & 0,46 & 4654,23 & 4,08 & $31,46(2,57)$ \\
\hline & $16^{\circ}$ & 6 & 4 & 1,38 & 0,47 & 4718,15 & 3,04 & $30,73(3,17)$ \\
\hline & $17^{\circ}$ & 1 & 3 & 1,16 & 0,45 & 4542,47 & 2,37 & $32,23(2,65)$ \\
\hline & $18^{\circ}$ & 5 & 3 & 1,81 & 0,45 & 4619,52 & 2,91 & $31,20(3,18)$ \\
\hline
\end{tabular}

${ }^{(1)}$ Valores entre parênteses indicam a média da relação SG para cada material genético. Idade, em anos; CA, relação cerne/alburno; DBM, densidade básica da madeira $\left(\mathrm{g} \mathrm{cm}^{-3}\right)$; PCSM, poder calorífico superior da madeira $\left(\mathrm{kcal} \mathrm{kg}^{-1}\right)$; EXT, teor de extrativos da madeira (\%); LT, teor de lignina total $(\%)$.
Com base nesses grupos, pode-se dizer que os materiais com maior densidade básica da madeira, maior idade e maior teor de extrativo obtiveram os melhores índices de qualidade. Neste caso, o aumento da idade desempenhou um papel fundamental, ao condicionar o aumento da DBM (Ferreira et al., 1979; Panshin \& Zeeuw, 1980) - também observado no presente trabalho - e o aumento no teor de extrativos, pela formação do cerne ocorrida com o envelhecimento da madeira. A formação do cerne é acompanhada da deposição de extrativos (Raven et al., 1996).

De acordo com Santos (2010) a lignina é um componente desejável na conversão da madeira em carvão, e seu teor e tipo são parâmetros importantes porque, em geral, espera-se que a conversão de madeira em carvão vegetal aumente com o aumento da proporção de lignina total e a diminuição da relação siringil/guaiacil, uma vez que isso promoveria maior

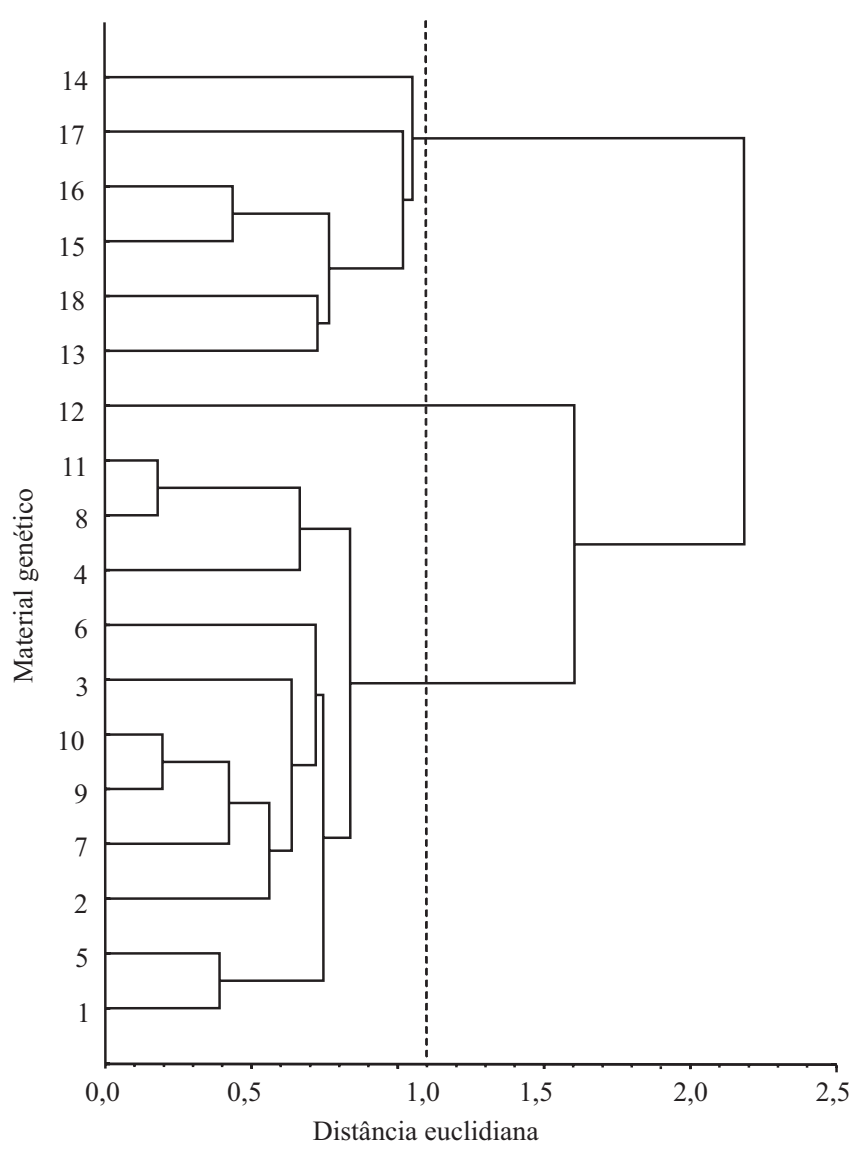

Figura 1. Agrupamento em função da qualidade da madeira quanto à produção de carvão vegetal, obtido pelo método de ligação simples com uso da distância euclidiana. 
resistência à degradação térmica, pela presença de estruturas mais condensadas. Portanto, esperava-se que o teor de lignina total tivesse influência positiva sobre a qualidade do carvão vegetal, o que, no entanto, não foi observado. A relação siringil/guaiacil (SG), apresentada junto aos valores de lignina total na Tabela 5, teve grande influência sobre o ranqueamento dos clones, pois os clones do primeiro grupo apresentaram menores valores da relação do que os do terceiro. Esse resultado é coerente, uma vez que quanto menor a relação $\mathrm{SG}$, maior a quantidade de lignina do tipo guaiacil, que apresenta estrutura mais condensada, o que conferiria maior resistência à degradação térmica (Gomide et al., 2005).

Lobão et al. (2011) aplicou estatística multivariada, para agrupar e identificar o potencial de uso de 12 espécies florestais (nativas e exóticas), a partir das propriedades anatômicas, físicas e químicas da madeira. Os autores concluíram que a análise de componentes principais foi mais eficiente do que o teste de Scott-Knott (estatística univariada), no agrupamento das espécies e na recomendação quanto ao uso da madeira. Entre as características avaliadas pelos autores, as mais importantes para o agrupamento das espécies foram: espessura da parede da fibra, densidade básica e teor de extrativos totais.

Confirma-se, com este trabalho, a importância de técnicas multivariadas para avaliação das variações na madeira que ocorrem nos diferentes clones de Eucalyptus, especialmente em razão da idade. Essas variações dificultam a seleção de materiais mais adequados para a produção de carvão vegetal, e a especificação das variáveis de madeira relacionadas favoravelmente à qualidade do carvão vegetal, bem como o agrupamento de materiais com qualidades semelhantes, pode facilitar os programas de melhoramento voltados à produção de madeira com fins energéticos (Adorno \& Garcia, 2003).

\section{Conclusões}

1. A estatística multivariada é eficiente na avaliação da qualidade da madeira para a produção de carvão vegetal.

2. Os materiais de melhor qualidade para uso energético são os que possuem maior densidade básica da madeira, que por sua vez aumenta com a idade.

3. A densidade aparente e o rendimento gravimétrico em carvão vegetal são as variáveis mais influenciadas pelas variáveis da madeira.
4. As características da madeira podem ser utilizadas para se inferir sobre as características do carvão vegetal.

\section{Agradecimentos}

Ao Conselho Nacional de Desenvolvimento Científico e Tecnológico (CNPq) e Fundação de Amparo a Pesquisa do Estado de Minas Gerais, pelo financiamento do projeto e pelas bolsas concedidas; à Gerdau S.A., pela disponibilização do material.

\section{Referências}

ADORNO, M. de F.C.; GARCIA, J.N. Correlações lineares entre as principais características tecnológicas da madeira de clones de Eucalyptus grandis e Eucalyptus urophylla. Scientia Forestalis, n.63, p.44-53, 2003.

ASSOCIAÇÃO BRASILEIRA DE NORMAS TÉCNICAS. NBR 8112: carvão vegetal: análise imediata: método de ensaio. Rio de Janeiro: ABNT, 1986. 8p.

ASSOCIAÇÃO BRASILEIRA DE NORMAS TÉCNICAS. NBR 8633: carvão vegetal: determinação do poder calorífico: método de ensaio. Rio de Janeiro: ABNT, 1984. 13p.

CASTRO, A.F.N.M. Efeito da idade e de materiais genéticos de Eucalyptus sp. na madeira e carvão vegetal. 2011. 98p. Dissertação (Mestrado) - Universidade Federal de Viçosa, Viçosa.

FERREIRA, C.A.; FREITAS, M. de; FERREIRA, M. Densidade básica da madeira de plantações comerciais de eucaliptos, na região de Mogi-Guaçu (SP). IPEF, n.18, p.106-117, 1979.

GOLDSCHIMID, O. Ultraviolet spectra. In: SARKANEN, K.V.; LUDWIG, C.H. (Ed.). Lignins: occurrence, formation, structure and reactions. New York: J. Wiley, 1971. p.241-266.

GOMIDE, J.L.; COLODETTE, J.L.; OLIVEIRA, R.C. de; SILVA, C.M. Caracterização tecnológica, para produção de celulose, da nova geração de clones de Eucalyptus no Brasil. Revista Árvore, v.29, p.129-137, 2005. DOI: 10.1590/S0100-67622005000100014.

GOMIDE, J.L.; DEMUNER, B.J. Determinação do teor de lignina em material lenhoso: método Klason modificado. O Papel, v.47, p.36-38, 1986.

GOULART, M.; HASELEIN, C.R.; HOPPE, J.M.; FARIAS, J.A.; PAULESKI, D.T. Massa específica básica e massa seca de madeira de Eucalyptus grandis sob o efeito do espaçamento de plantio e da posição axial no tronco. Ciência Florestal, v.13, p.167-175, 2003.

LIN, S.Y.; DENCE, C.W. Methods in lignin chemistry. Berlin: Springer Verlag, 1992. 578p. DOI: 10.1007/978-3-642-74065-7.

LOBÃO, M.S.; CASTRO, V.R. de; RANGEL, A.; SARTO, C.; TOMAZELLO FILHO, M.; SILVA JÚNIOR, F.G. da; CAMARGO NETO, L. de; BERMUDEZ, M.A.R.C. Agrupamento de espécies florestais por análises univariadas e multivariadas das características anatômica, física e química das suas madeiras. Scientia Forestalis, v.39, p.469-477, 2011. 
MINGOTI, S.A. Análise de dados através de métodos de estatística multivariada: uma abordagem aplicada. Belo Horizonte: Ed. UFMG, 2005. 297p.

PANSHIN, A.J.; ZEEUW, C. de. Textbook of wood technology. $4^{\text {th }}$ ed. New York: $1980.722 p$.

PEREIRA, B.L.C.; OLIVEIRA, A.O.; CARVALHO, A.M.M.L.; CARNEIRO, A. de C.O.; SANTOS, L.C.; VITAL, B.R. Quality of wood and charcoal from Eucalyptus clones for ironmaster use. International Journal of Forestry Research, v.2012, p.1-8, 2012. DOI: $10.1155 / 2012 / 523025$.

PROTÁSIO, T. de P.; COUTO, A.M.; REIS, A.A. dos; TRUGILHO, P.F. Seleção de clones de Eucalyptus para a produção de carvão vegetal e bioenergia por meio de técnicas univariadas e multivariadas. Scientia Forestalis, v.41, p.15-28, 2013.

RAVEN, P.H.; EVERT, R.F.; EICHHORN, S.E. Biologia vegetal. 5.ed. Rio de Janeiro: Guanabara Koogan, 1996. 738p.

RIBEIRO, F. de A.; ZANI FILHO, J. Variação da densidade básica da madeira em espécies/procedências de Eucalyptus spp. IPEF, n.46, p.76-85, 1993.

RIBEIRO, J.F.; WALTER, B.M.T. Fitofisionomias do Bioma Cerrado. In: SANO, S.M.; ALMEIDA, S.P. (Ed.). Cerrado: ambiente e flora. Planaltina: Embrapa-CPAC, 1998. p.89-166.

SANTOS, R.C. Parâmetros de qualidade da madeira e do carvão vegetal de clones de eucalipto. 2010. 173p. Tese (Doutorado) Universidade Federal de Lavras, Lavras.
SILVA, J. de C.; OLIVEIRA, J.T. da S.; TOMAZELLO FILHO, M.; KEINERT JÚNIOR, S.; MATOS, J.L.M. de. Influência da idade e da posição radial na massa específica da madeira de Eucalyptus grandis Hill ex. Maiden. Revista Floresta, v.34, p.13-22, 2004.

SOUZA, P.M. de. Modernização e mudanças estruturais na agricultura brasileira, 1970 a 1995. 2000. 287p. Tese (Doutorado) - Universidade Federal de Viçosa, Viçosa.

SOUZA, P.M. de; LIMA, J.E. de. Intensidade e dinâmica da modernização agrícola no Brasil e nas unidades da Federação. Revista Brasileira de Economia, v.57, p.795-824, 2003. DOI: 10.1590/S0034-71402003000400007.

TAPPI TECHNICAL DIVISIONS AND COMMITTEES. TAPPI test methods: T $257 \mathrm{~cm}-85$ : sampling and preparing wood for analysis. Atlanta: Tappi Technical Divisions and Committees, 1985. 5p.

TAPPI TECHNICAL DIVISIONS AND COMMITTEES. TAPPI test methods: T $264 \mathrm{~cm}-97$ : preparation of wood for chemical analysis. Atlanta: Tappi Technical Divisions and Committees, 1997. 3p.

TRUGILHO, P.F.; LIMA, J.T.; MORI, F.A. Correlação canônica das características químicas e físicas da madeira de clones de Eucalyptus grandis e Eucalyptus saligna. Cerne, v.9, p.66-80, 2003.

VITAL, B.R. Métodos de determinação de densidade da madeira. Viçosa: Sociedade de Investigações Florestais, 1984. 21p. (SIF. Boletim técnico, 1).

Recebido em 4 de outubro de 2012 e aprovado em 31 de maio de 2013

Pesq. agropec. bras., Brasília, v.48, n.6, p.627-635, jun. 2013 DOI: 10.1590/S0100-204X2013000600008 